\begin{tabular}{ll} 
Journal of Environmental \\
Analysis and Progress \\
ISSN: $2525-815 \mathrm{X}$ & Journal homepage: $\underline{\text { www.jeap.ufrpe.br/ }}$ \\
\hline
\end{tabular}

\title{
Análise estatística da velocidade do vento em Petrolina-PE utilizando as distribuições Weibull e a Burr
}

\section{Statistical analysis of the Wind speed in Petrolina-PE using the Weibull and Burr distributions}

Fábio Sandro dos Santos ${ }^{\mathrm{a}}$, Kerolly Kedma Felix do Nascimento ${ }^{\mathrm{a}}$, Elielma Santana de Jesus ${ }^{\mathrm{a}}$, Jader da Silva Jale ${ }^{a}$, Tatijana Stosic ${ }^{a}$, Tiago Alessandro Espínola Ferreira ${ }^{\mathrm{a}}$

${ }^{\text {a } U n i v e r s i d a d e ~ F e d e r a l ~ R u r a l ~ d e ~ P e r n a m b u c o-U F R P E, ~ C a m p u s ~ D o i s ~ I r m a ̃ o s, ~ R u a ~ D o m ~ M a n o e l ~ d e ~ M e d e i r o s, ~ S / N º, ~ D o i s ~}$ Irmãos, Recife, Pernambuco, Brasil. CEP: 52171-900. E-mail: fabio.sandropb@gmail.com, kerollyfn@gmail.com, elielmas93@gmail.com, jsj_ce@yahoo.com.br, tastosic@gmail.com, taef.first@ gmail.com.

\begin{tabular}{|c|}
\hline A RT I CLE IN F O \\
\hline Recebido 20 Jul 2018 \\
\hline Aceito 30 Jan 2019 \\
\hline Publicado 31 Jan 2019 \\
\hline
\end{tabular}

\begin{abstract}
A B S T R A C T
For investment deliberation and consequent installation of a wind farm in each region, analyzes with probability density functions of distribution models can be carried out that are adjusted to the local wind speed data. Therefore, in order to assist in the optimal decision-making, it is necessary to choose the model that presents the best fit in relation to the others. The objective of this study is to verify the quality of the adjustments made by the Weibull distributions with two parameters and Burr to the historical series of wind speed in Petrolina-PE, 01/01/2015 to 12/31/2016. The parameters of the distributions were estimated with the Maximum Likelihood Method, which has reached optimal values in relation to other methods of parameter estimation. The Akaike Information Criterion, Bayesian Information Criterion, Anderson-Darling statistic, and Mean Absolute Percent Error and Mean Absolute Standard Deviation estimates were used to evaluate the goodness of distributions adjustments. In addition, the predominant direction of the winds was observed through the Wind Rose. The results showed that Weibull, with two parameters, provided a better modeling of the analyzed data and the predominant direction of the observed winds was to the southeast, varying between $\sim 105^{\circ}$ and $\sim 135^{\circ}$ and mean velocity of $8.4 \mathrm{~m} \cdot \mathrm{s}^{-1}$. According to the classification of the National Renewable Energy Laboratory, the region studied achieved a splendid assessment for the viability of wind energy generation. Therefore, it is recommended to use the Weibull distribution with two parameters for further investigation.
\end{abstract}

Keywords: Adjustment, energy, modeling, wind potential, Wind rose.

\section{R E S U M O}

Para deliberação do investimento e consequente instalação de parque eólico em uma dada região, podem ser realizadas análises com funções densidade de probabilidade de modelos de distribuição que se ajustam aos dados de velocidade do vento da localidade. Por isso, para auxiliar a tomada de decisão ideal, faz-se necessária a escolha do modelo que apresente o melhor ajuste em relação aos demais. O objetivo deste estudo foi verificar a qualidade dos ajustes realizados pelas distribuições Weibull com dois parâmetros e Burr para a série histórica de velocidade do vento em Petrolina-PE, de 01/01/2015 a 31/12/2016. Os parâmetros das distribuições foram estimados com o Método da Máxima Verossimilhança, o qual tem alcançado valores ótimos em relação a outros métodos de estimativa de parâmetros. O Critério de Informação de Akaike, o Critério de Informação Bayesiano, a estatística de Anderson-Darling e as acurácias de Média Percentual Absoluta do Erro e Desvio Padrão Absoluto da Média foram adotados para avaliação da bondade dos ajustes das distribuições. Além disso, a direção predominante dos ventos foi observada por meio da Rosa dos Ventos. Os resultados indicam que a Weibull, com dois parâmetros, forneceu melhor modelagem aos dados analisados e a direção predominante dos 
ventos observada foi a sudeste, com variação entre $\sim 105^{\circ} \mathrm{e} \sim 135^{\circ}$ e velocidade média de $8,4 \mathrm{~m} \cdot \mathrm{s}^{-1}$. Segundo classificação do National Renewable Energy Laboratory, a região estudada alcançou avaliação esplêndida para a viabilidade de geração de energia eólica. Portanto, recomenda-se a utilização da distribuição Weibull com dois parâmetros para investigações posteriores.

Palavras-Chave: Ajuste, energia, modelagem, potencial eólico, Rosa dos ventos.

\section{Introdução}

No atual cenário mundial, onde buscam-se fontes renováveis de energia, o Brasil tem obtido destaque devido a variedade de recursos naturais disponíveis em seu território. Neste âmbito, destacam-se os ventos como potenciais produtores de energia, pois os mesmos não emitem gases poluentes na atmosfera, estão em constante processo de renovação e, com sua utilização, reduz-se a necessidade de utilização de combustíveis fósseis. Entre outras vantagens para a sociedade, pode-se mencionar a geração de emprego e investimento nas localidades onde são instalados os parques eólicos. Além disso, a relação custo-benefício da energia eólica pode ser considerada das melhores, em comparação a outras fontes de energia, além de não exigir frequência na manutenção de seus equipamentos (PE, 2018).

A região Nordeste do Brasil apresenta rajadas de vento com altas frequências em suas velocidades médias (Lopeset al., 2017), o que proporciona um grande potencial para $\mathrm{o}$ aproveitamento de produção de energia elétrica. Em destaque, os estados da Bahia, do Ceará, do Rio Grande do Norte, do Rio Grande do Sul e do Piauí, em sua grande maioria contidos na região Nordeste, concentram grande quantidade dos parques eólicos instalados no país, gerando mais de 10GWs de capacidade instalada de energia eólica (ABEeolica, 2018).

Para a escolha de locais adequados à instalação de um sistema com capacidade de aproveitamento de energia eólica faz-se necessário o estudo da frequência e da intensidade em que os ventos ocorrem (Zaharim et al., 2009). Neste sentido, pesquisadores, utilizando a estatística, podem desenvolver procedimentos para a compreensão do comportamento dos ventos, com o auxílio das distribuições probabilísticas que melhor se adequam a esses conjuntos de dados. Entre as diversas distribuições existentes, aquelas que são comumente empregadas, e se encontram abundantemente na literatura, sobre modelagem de regimes de ventos são: a Burr, a Gama, a Erlang, aWeibull e a Lognormal (Masseran et al., 2012; Brano et al., 2011).

Dentre os diferentes métodos que podem ser aplicados para a estimativa de parâmetros e sabendo que os mesmos podem apresentar diferentes desempenhos de acordo com cada conjunto de dados utilizado e suas aplicações (Ozawa, 2017), é importante a escolha adequada do método empregado.

Diante do exposto, o objetivo do estudo é identificar uma melhor distribuição probabilística para a modelagem da velocdade dos ventos, sob diferentes condições ambientais.

\section{Material e Métodos}

Área de estudo

O estudo foi realizado na cidade de Petrolina-PE (Figura 1), localizada no sertão pernambucano, no Nordeste brasileiro, próxima ao rio São Francisco, com população estimada de, aproximadamente, 343.219 habitantes, em 2017 (IBGE, 2018).

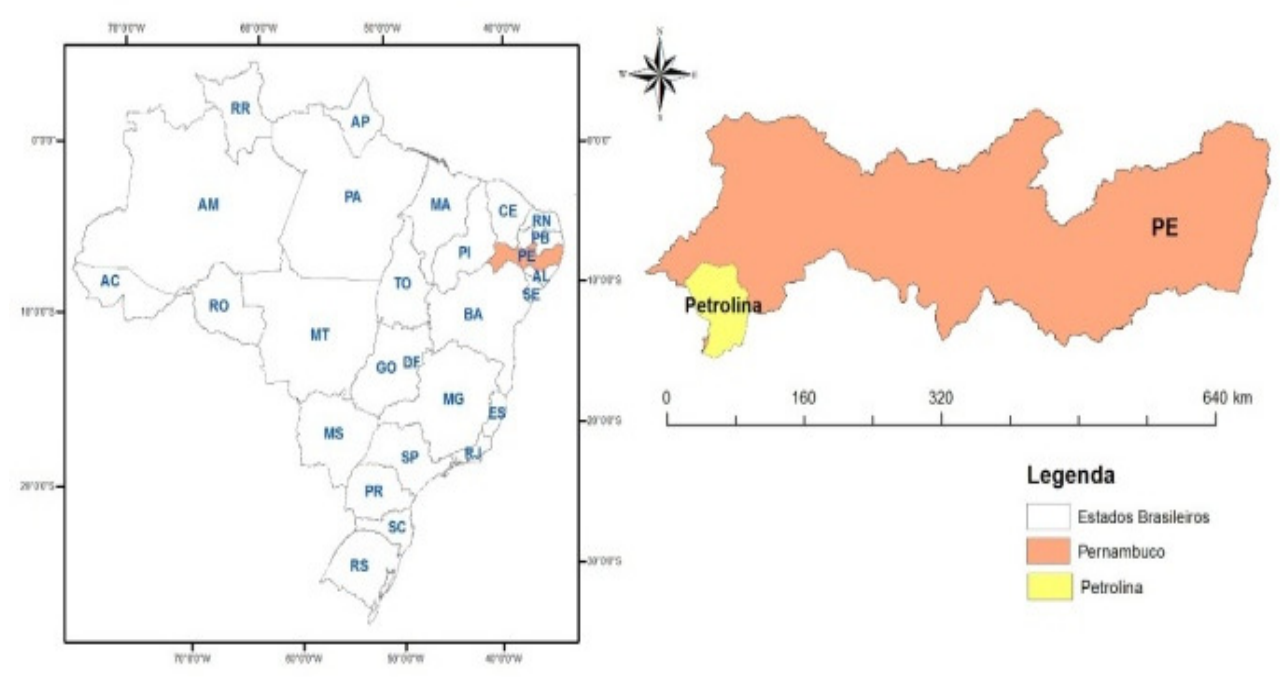

Figura 1. Localização do município de Petrolina-PE. 


\section{Dados}

Os dados de velocidade do vento utilizados para a realização deste estudo foram obtidos no Instituto Nacional de Meteorologia (INMET, 2018), para o período compreendido entre 01/01/2015 e 31/12/2016, com registro a cada três horas. Esses dados foram obtidos da estação meteorológica localizada nas coordenadas geográficas de longitude -40,367, latitude -9,150 e altitude de $366 \mathrm{~m}$, na cidade de Petrolina-PE.

\section{Rosa dos Ventos}

A Rosa dos Ventos exibe a predominância dos ventos em cada direção, com suas respectivas velocidades. Em geral, encontra-se dividida em setores (Keyhani et al., 2010; Pishgar-Komleh et al., 2015). Através do estudo adequado da Rosa dos Ventos é possível posicionar os aerogeradores de modo mais eficiente para captação dos ventos e, consequentemente, geração de energia.

\section{Modelo de distribuição Weibull-2p}

A função densidade de probabilidade (FDP) da Weibull-2p vem sendo usada repetidamente na verificação do potencial eólico e da viabilidade de instalação de parques eólicos (Dokur et al., 2016; Akgül et al., 2016; Rosa Filho et al., 2018). A FDP da Weibull-2p pode ser definida conforme a expressão matemática da Equação 1.

$$
f_{W}(x ; \gamma, \alpha)=\frac{\gamma}{\alpha}\left(\frac{x}{\alpha}\right)^{\gamma-1} \exp \left[-\left(\frac{x}{\alpha}\right)^{\gamma}\right] x ; \gamma, \alpha>0,
$$

onde $f_{W}(x ; \gamma, \alpha)$ é a probabilidade calculada da velocidade do vento; $x, \gamma$ e $\alpha$ são parâmetros de forma e escala, respectivamente.

Além disso, foi usada a Função de Distribuição Acumulada (FDA) para determinar o potencial de velocidade do vento na região em estudo. A FDA da distribuição Weibull-2p está definida na Equação 2.

$$
F_{W}(x ; \gamma, \alpha)=1-\exp \left[-\left(\frac{x}{\alpha}\right)^{\gamma}\right] x ; \gamma, \alpha>0
$$

\section{Modelo de distribuição Burr}

As aplicações da distribuição Burr aos dados de velocidade de vento são mais recentes, em comparação às realizadas pela distribuição Weibull-2p. Entretanto, os resultados obtidos têm se mostrado bastante promissores e seu modelo tem sido cada vez mais empregado no estudo dos potenciais eólicos de regiões propícias à instalação de usinas que permitam o aproveitamento dos ventos para geração de energia (Brano et al., 2011; Allouhi et al., 2017).
As Equações 3 e 4 expressam a FDP e a FDA da distribuição Burr, respectivamente,

$$
\begin{aligned}
& f_{B}(x ; \gamma, \alpha, c)=\frac{\alpha \gamma x^{\alpha-1}}{c^{\alpha}\left[1+\left(\frac{x}{c}\right)^{\alpha}\right]^{1+\gamma}} \quad x, \gamma, \alpha, c>0 \\
& F_{B}(x ; \gamma, \alpha, c)=1-\left(1+x^{\alpha}\right)^{-\gamma} \quad x, \gamma, \alpha, \mathrm{c}>0,
\end{aligned}
$$

onde $f_{B}(x ; \gamma, \alpha, c)$ expressa a probabilidade da velocidade do vento; $x, \alpha$ e $c$ são os parâmetros de escala e $\gamma$ o parâmetro de forma.

\section{Método de Máxima Verossimilhança (MMV)}

Para a estimação dos parâmetros de forma e de escala dos modelos adotados neste trabalho existe uma variedade de métodos. Contudo, devido a sua imensa aplicabilidade estatística, adotou-se o Método de Máxima Verossimilhança (Alavi et al., 2016).

Esse método é considerado como um dos melhores para a estimação de parâmetros das distribuições de probabilidade. Em sua busca, o estimador de máxima verossimilhança é o valor que maximiza a função de verossimilhança. Matematicamente, pode-se expressá-la segundo a Equação 5.

$$
L\left(\theta ; x_{1}, \ldots, x_{n}\right)=\prod_{i=1}^{n} f\left(x_{i} ; \theta\right)
$$

Critérios de seleção do ajuste das distribuições Weibull-2p e Burr e medidas de acurácia

$\mathrm{Na}$ verificação da bondade dos ajustes realizados pelos modelos das distribuições Weibull-2p e Burr, à base de dados, foram utilizados o Critério de Informação de Akaike (AIC), o Critério de Informação Bayesiano (BIC) e as estatísticas Anderson-Darling (AD), Média Percentual Absoluta do Erro (MAPE) e o Desvio Padrão Absoluto da Média (MAD).

Considerando os resultados após aplicação dos critérios AIC e BIC, quanto menores os valores encontrados para essas medidas, melhores são os ajustes realizados pelas distribuições. $\mathrm{O}$ teste estatístico de Anderson-Darling pode ser usado para definir a melhor adequabilidade de uma determinada distribuição de probabilidade em relação a outra distribuição, em um conjunto de dados (Yilmaz \& Çelik, 2008). Para as medidas de acurácia MAPE e MAD, além da avaliação dos erros associados aos ajustes, quanto mais próximo de zero for o valor da estimativa encontrada, melhor é o ajuste da distribuição na série histórica de velocidade do vento.

A Tabela 1 exibe as expressões matemáticas para cada uma dessas estatísticas. 
Tabela 1. Definições estatísticas dos critérios de seleção de modelos e das medidas de acurácia.

\section{Estatística Fórmula matemática}

$$
\begin{aligned}
& \text { AIC } \quad A I C=-2 \log (L)+2[(p+1) \\
& +1] \\
& B I C=-2 \log (L)+[(p+1) \\
& +1] \log (n) \\
& A D^{2}=-N-S
\end{aligned}
$$

MAPE

$$
\text { MAPE }=\frac{1}{\mathrm{n}} \sum_{\mathrm{i}=1}^{\mathrm{n}}\left|\frac{y_{i}-\hat{y}}{\hat{y}}\right| * 100
$$

MAD

$$
\mathrm{MAD}=\frac{1}{\mathrm{n}} \sum_{\mathrm{i}=1}^{\mathrm{n}}\left(\frac{y_{i}-\hat{y}}{\hat{y}}\right) * 100
$$

$S=\sum_{i=1}^{n} \frac{(2 i-1)}{N}\left[\log F\left(y_{i}\right)+\log \left(1-F\left(y_{(N+1-1)}\right)\right)\right](6)$ onde $y_{i}$ representa as observações de velocidade do vento, $\widehat{y}$ é cada valor estimado, $n$ é a quantidade de observações de velocidade do vento, $L$ é função de verossimilhança do modelo e $p$ é a quantidade de parâmetros.

\section{Resultados}

A aplicação da estatística descritiva permitiu caracterizar o comportamento da principal variável em estudo, velocidade do vento na cidade de Petrolina-PE, conforme a Tabela 2. Observa-se que o Coeficiente de Variação $(\mathrm{CV})$ referente à série examinada foi de $41 \%$ (Tabela 2), indicando, portanto, que os dados são bastante dispersos em torno da sua média.

Os resultados mostram que o município de Petrolina-PE apresenta um valor médio de velocidade do vento em torno de $8,4 \mathrm{~m} \cdot \mathrm{s}^{-1}$. A série histórica dos dados pode ser visualizada através da Figura 2

Tabela 2. Estatística descritiva da série temporal de velocidade do vento em Petrolina-PE.

\begin{tabular}{llllllllll}
\hline Min & $\boldsymbol{Q}_{\mathbf{1}}$ & Med & Méd & $\boldsymbol{Q}_{\mathbf{3}}$ & Max & SD & CV & $\boldsymbol{a}_{\mathbf{3}}$ & $\boldsymbol{a}_{\mathbf{4}}$ \\
\hline 0.4 & 5.7 & 8.3 & 8.4 & 10.9 & 19.8 & 3.4 & 0.41 & 0.21 & 2.38 \\
\hline
\end{tabular}

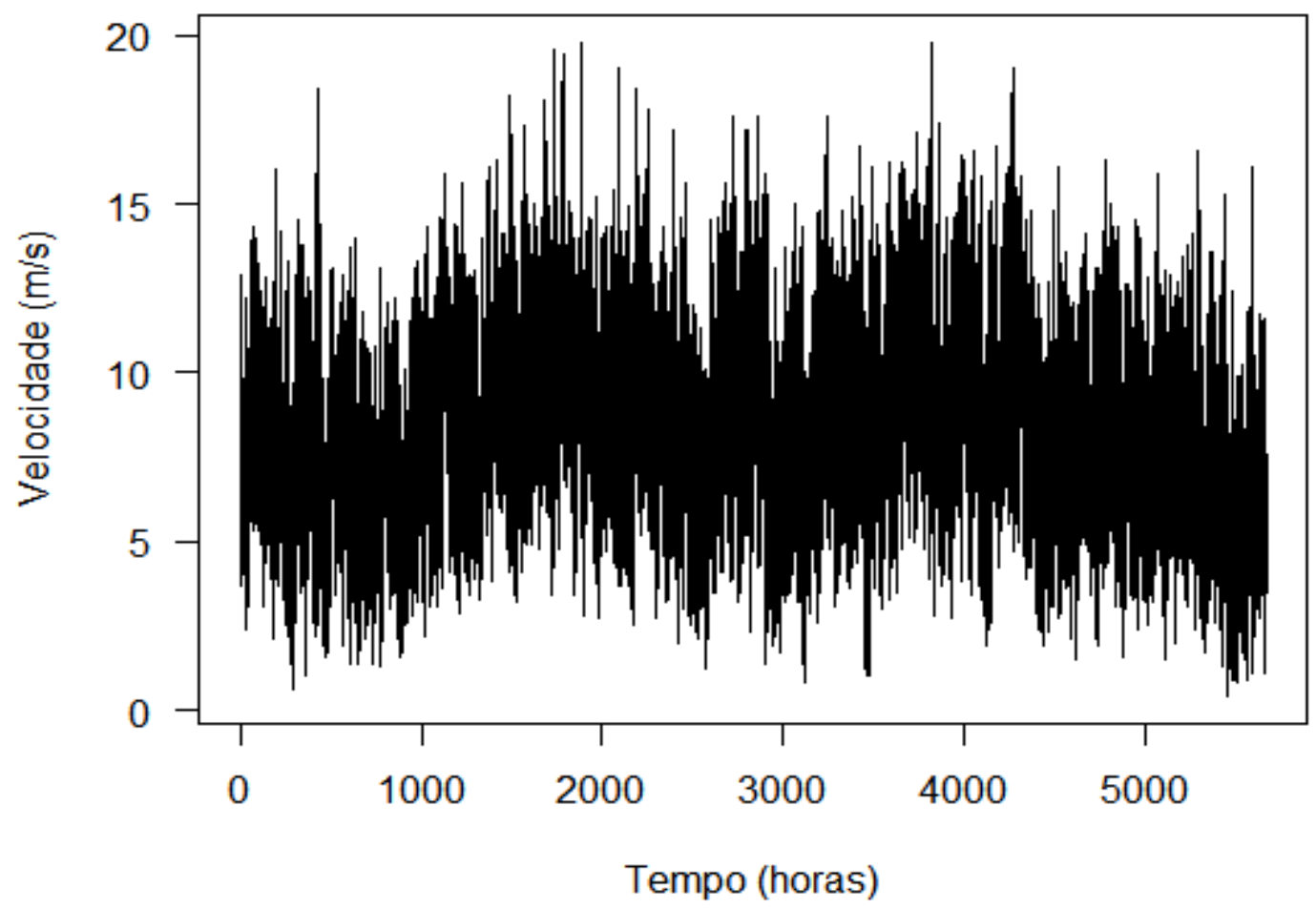

Figura 2. Série de velocidade do vento, de três em três horas, no período de 01/01/2015 a 31/12/2016, em Petrolina-PE.

Com a Rosa dos Ventos (Figura 3) foi possível verificar a variabilidade da direção predominante do vento no período analisado. $\mathrm{O}$ diagrama está dividido em parcelas de 12 setores, compostos por ângulos de $15^{\circ}$ cada, no sentido horário. Verifica-se que a direção predominante é a direção sudeste, com variação entre $\sim 105^{\circ} \mathrm{e} \sim 135^{\circ}$. 


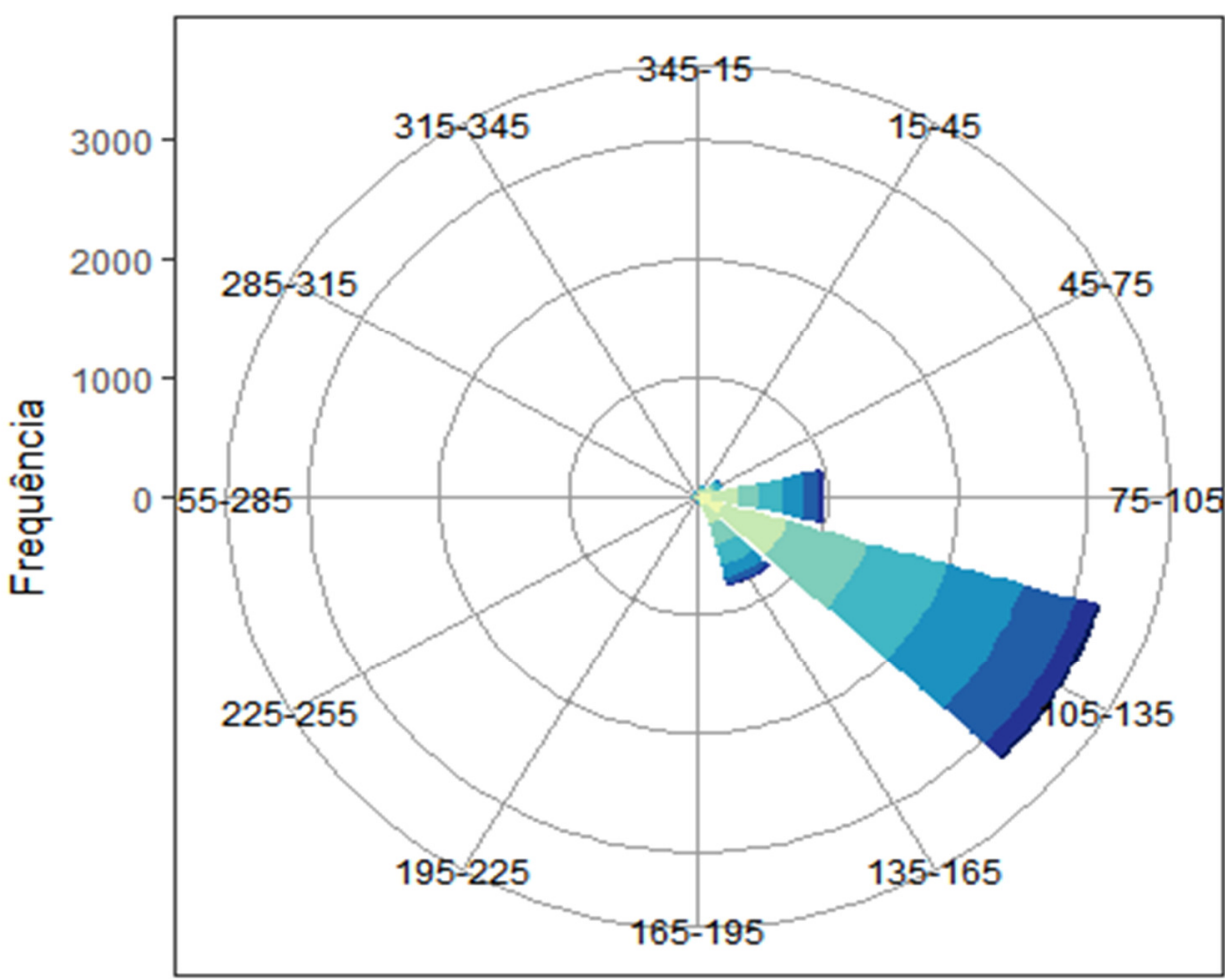

Velocidade $(\mathrm{m} / \mathrm{s})$

\begin{tabular}{|l|l|}
\hline $16-18$ \\
$14-16$ \\
$12-14$ \\
$10-12$ \\
$8-10$ \\
$6-8$ \\
$4-6$ \\
$2-4$ \\
$0-2$ \\
\hline
\end{tabular}

Figura 3. Rosa dos Ventos indicando a variabilidade da direção predominante do vento em Petrolina-PE.

O cálculo dos parâmetros das distribuições

Weibull-2p e Burr permitiu uma melhor modelagem dos dados amostrais. Os resultados das FDP's e FDA's encontram-se nas Figuras 4 e 5.

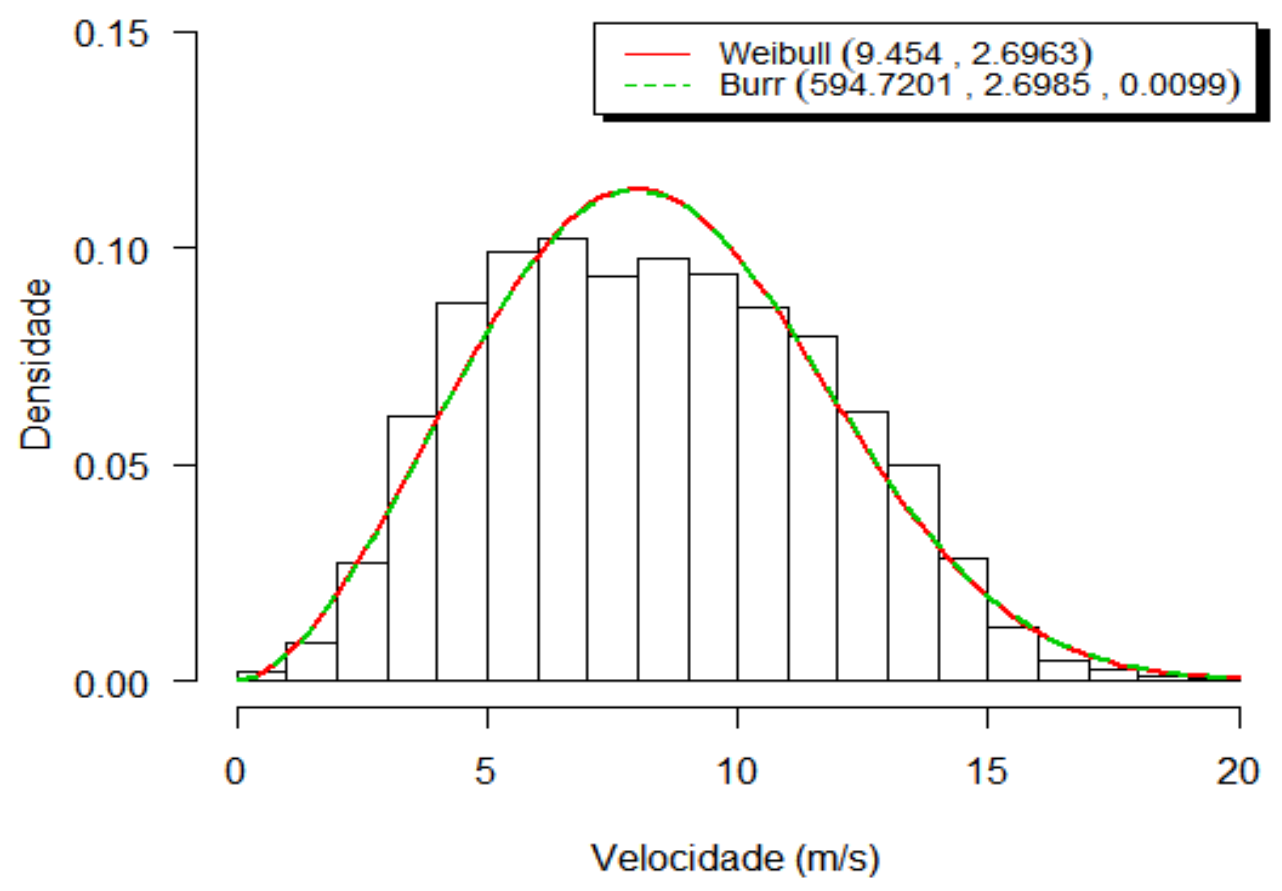

Figura 4. Ajustes da função densidade de probabilidade da Weibull-2p e da Burr. 


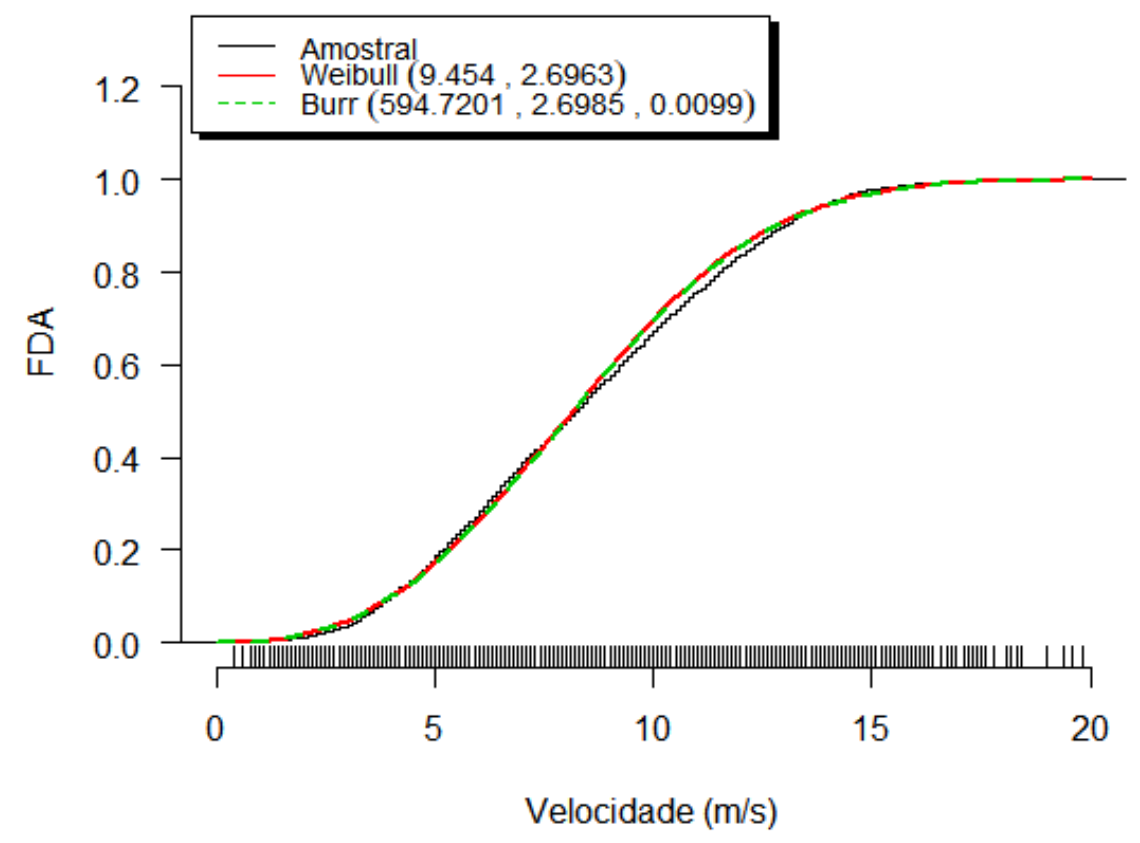

Figura 5. Função de distribuição acumulada dos dados amostrais, das distribuições Weibull-2p e da Burr ajustadas.

Para a validação dos resultados, obtidos graficamente, e análise, quanto à distribuição com melhor ajuste aos dados, foi aplicado o teste estatístico de Anderson-Darling (Stephens, 1986), apresentado na Tabela 3.

Tabela 3. Estatística de Anderson-Darling para avaliação do ajuste das distribuições Weibull-2p e Burr aos dados analisados.

\begin{tabular}{lcc}
\hline Distribuição & AD & p-value \\
\hline Weibull-2p & 4.96 & 0.003 \\
Burr & 3.25 & 0.0206 \\
\hline
\end{tabular}

A partir dos valores obtidos após aplicação da estatística, foi detectado que eles são bem próximos. Portanto, foram utilizados critérios de seleção de modelos (AIC e BIC) e medidas de acurácia dos erros (MAPE e MAD) para auxiliar na decisão sobre qual delas é a melhor distribuição para o ajuste, conforme resultados indicados na Tabela 4.

Tabela 4. Critérios de seleção e acurácias para o ajuste das distribuições Weibull-2p e Burr para o estudo da velocidade do vento em Petrolina-PE.

\begin{tabular}{lcc}
\hline $\begin{array}{l}\text { Critérios e } \\
\text { erros }\end{array}$ & Weibull-2p & Burr \\
\hline AIC & 29611,0305 & 29613,7014 \\
BIC & 29624,3093 & 29633,6196 \\
MAPE & 5,1803 & 5,2531 \\
MAD & 0,0144 & 0,0144 \\
\hline
\end{tabular}

\section{Discussão}

A distribuição Burr, ao nível de significância de 0,05 , pelo teste de AndersonDarling, apresenta indícios de um melhor ajuste à série de dados analisada, em comparação à distribuição Weibull-2p, em concordância com estudos anteriores (Abbas et al., 2012; Mert \& Karakus, 2015).

Abbas et al. (2012) ajustaram as distribuições Gama, Weibull, Lognormal, Rayleigh, Burr e Frechet aos dados diários de velocidade do vento, em Islamabade, Paquistão, entre 2001 e 2003, utilizando três testes de qualidade de ajuste, ao nível de significância de 5\% (qui-quadrado, Kolmogorov-Smirnov e AndersonDarling). Os autores concluíram que as distribuições Gama, Lognormal e Burr apresentaram melhor ajuste em comparação com as outras três.

Mert \& Karakus (2012) analisaram, estatisticamente, o potencial de energia eólica da cidade de Antáquia, no sudeste da Turquia, entre 2002-2009, e testaram o ajuste das distribuições Burr-4p, Gama generalizada-3p e Weibull utilizando $R^{2}, \quad R M S E$, qui-quadrado $\mathrm{e}$ Kolmogorov-Smirnov. Esses autores concluíram que a distribuição Burr foi mais adequada que a Gama generalizada ou Weibull.

Entretanto, nesse estudo, a análise estatística, para ambos os testes, mostrou valores bem próximos. Por isso, foram calculados os critérios de seleção e as acurácias, com o objetivo de fazer uma escolha mais acertada quanto ao modelo que melhor se ajustasse aos dados. Assim, 
verificou-se que o AIC, o BIC e o MAPE apresentaram menores valores para a distribuição Weibull-2p; o MAD foi igual para ambas as distribuições. Isso indica que, para o conjunto de dados de Petrolina-PE, no período analisado, a distribuição Weibull-2p consegue modelar melhor as informações, em detrimento da distribuição Burr.

Em estudos recentes, realizados em diferentes localidades do Brasil (Grah et al., 2014), com destaque especial para aqueles no Nordeste brasileiro (Santos \& Silva, 2016; Rocha et al., 2012), observa-se a utilização da distribuição Weibull com dois parâmetros e seu bom ajuste aos dados de velocidade do vento.

Segundo a classificação do National Renewable Energy Laboratory (NREL) e Santos et al. (2018), o valor encontrado para a velocidade média do vento, a $10 \mathrm{~m}$ da superfície, correspondendo à classe de vento 7, comparada ao valor médio de, aproximadamente, $8,4 \mathrm{~m} \cdot \mathrm{s}^{-1}$, obtido em Petrolina-PE, representando a descrição definida como "esplêndida", para a caracterização do potencial eólico na região analisada.

Dessa maneira, os resultados encontrados neste estudo, para o ajuste da distribuição Weibull$2 \mathrm{p}$, têm se mostrado coerentes, quando comparados aos encontrados na literatura.

\section{Conclusão}

As distribuições Weibull-2p e Burr apresentaram ajustes semelhantes à série de velocidade do vento em Petrolina-PE, no período estudado. Entretanto, pelos critérios de seleção aqui adotados, verificou-se que a distribuição Weibull-2p é mais indicada à modelagem dos dados analisados.

Constatou-se um valor médio de velocidade do vento, com uma classificação que apresenta indícios de que a localidade possui potencial para investigações mais aprofundadas e possível instalação de um parque eólico na região, tendo as pás dos aerogeradores voltadas na direção sudeste, a qual foi observada como a predominante dos ventos, com o auxílio da Rosa dos Ventos.

\section{Agradecimentos}

Os autores agradecem à Coordenação de Aperfeiçoamento de Pessoal de Nível Superior (CAPES), pelo apoio financeiro.

\section{Referências}

ABBAS, K.; ALAMGIR, K. S.; ALI, A., KHAN, D. M.; KHALIL, U. 2012. Statistical analysis of wind speed data in Pakistan. World Applied Sciences Journal, v. 18, n. 11, p. 1533-1539.
ABEEólica. $2018 . \quad$ Disponível em:http://abeeolica.org.br/noticias/brasil-chega-a13-gw-de-capacidade-instalada-de-energia-eolica/ Acesso em: 10-jul-2018.

AKGÜL, F. G.; ŞENOĞLU, B.; ARSLAN, T. 2016. An alternative distribution to Weibull for modeling the wind speed data: Inverse Weibull distribution. Energy Conversion and Management, v. 114 , p. 234-240.

ALAVI, O.; MOSTAFAEIPOUR, A.; QOLIPOUR, M. 2016. Analysis of hydrogen production from wind energy in the southeast of Iran. International Journal of Hydrogen Energy, v. 41, n. 34, p. 15158-15171.

ALLOUHI, A.; ZAMZOUM, O.; ISLAM, M. R.; SAIDUR, R.; KOUSKSOU, T.; JAMIL, A.; DEROUICH, A. 2017. Evaluation of wind energy potential in Morocco's coastal regions. Renewable and Sustainable Energy Reviews, v. 72, p. 311324.

BRANO, V. L.; ORIOLI, A.; CIULLA, G.; CULOTTA, S. 2011. Quality of wind speed fitting distributions for the urban area of Palermo, Italy. Renewable Energy, v. 36, n. 3, p. 1026-1039.

DOKUR, E.; KURBAN, M.; CEYHAN, S. 2016. Wind speed modelling using inverse Weibull distribution: a case study for Bilecik, Turkey. International Journal of Energy Applications and Technologies, v. 3, n. 2, p. 55-59.

GRAH, V. F.; PONCIANO, I. M.; BOTREL, T. A. 2014. Potential for wind energy generation in Piracicaba, SP, Brazil. Revista Brasileira de Engenharia Agrícola e Ambiental, v. 18, n. 5, p. 559-564.

IBGE. 2018. Disponível em: https://cidades.ibge.gov.br/brasil/pe/petrolina/pan orama. Acesso em: 19-jul-2018.

INMET. 2018. Instituto Nacional de Meteorologia. Disponível em: http://www.inmetgov.br. Acesso em: 09-Jun-2018.

KEYHANI, A.; GHASEMI-VARNAMKHASTI, M.; KHANALI, M.; ABBASZADEH, R. 2010. An assessment of wind energy potential as a power generation source in the capital of Iran, Tehran. Energy, v. 35, n. 1, p. 188-201.

LOPES, I.; DE CERQUEIRA JÚNIOR, E. P.; DE MELO, J. M. M.; LEAL, B. G. 2017. Potencial de 
geração de energia eólica no Submédio São Francisco. Journal of Environmental Analysis and Progress, v. 2, n. 3, p. 330-340.

MASSERAN, N.; RAZALI, A. M.; IBRAHIM, K. 2012. An analysis of Wind Power density derived from several Wind speed density functions: The regional assessment on wind power in Malaysia. Renewable and Sustainable Energy Reviews, v. 16, n. 8, p. 6476-6487.

MERT, I.; KARAKUŞ, C. (2015). A statistical analysis of wind speed data using Burr, generalized gamma, and Weibull distributions in Antakya, Turkey. Turkish Journal of Electrical Engineering \& Computer Sciences, v. 23, n. 6, p. 1571-1586.

OZAWA, M. T. 2017. Aplicação de parâmetros da distribuição de Weibull na análise do potencial energético de um microgerador eólico em cidades do estado do Paraná. Trabalho de Conclusão de Curso. Universidade Tecnológica Federal do Paraná.

PATEL, M. R. 1999. Wind and Solar Power Systems. Copyrighted material. 345p.

PE. 2018. Portal energia - energia renováveis. Disponível em: https://www.portalenergia.com/vantagens-desvantagens-da-energiaeolica/. Acesso em: 11-jul-2018.

PISHGAR-KOMLEH, S. H.; KEYHANI, A.; SEFEEDPARI, P. 2015. Wind speed and power density analysis based on Weibull and Rayleigh distributions (a case study: Firouzkooh county of Iran). Renewable and Sustainable Energy Reviews, v. 42, p. 313-322.

ROCHA, P. A. C.; DE SOUSA, R. C.; DE ANDRADE, C. F.; DA SILVA, M. E. V. 2012.
Comparison of seven numerical methods for determining Weibull parameters for Wind energy generation in the northeast region of Brazil. Applied Energy, v. 89, n. 1, p. 395-400.

ROSA FILHO, C. DE D.; PARISI, S. A.; DE ALBUQUERQUE SOARES, W. 2018. Avaliação da distribuição estatística e elaboração de modelo de regressão múltipla linear da velocidade média do vento. Journal of Environmental Analysis and Progress, v. 3, n. 1, p. 24-36.

SANTOS, D. V. G. DOS; LIRA, C. C. S.; LEAL JUNIOR, J. B. V. 2018. Avaliação do potencial eólico da região do Vale do Jaguaribe no semiárido cearense utilizando modelagem numérica regional. Journal of Environmental Analysis and Progress, v. 3, n. 1, p. 69-74.

SANTOS, A. T. S.; SILVA, C. M. S. 2016. Assessment of Wind resources in two parts of Northeast Brazil with the use of numerical models. Meteorological Applications, v. 23, n. 4, p. 563-573.

STEPHENS, M. A. 1986. Tests Based on EDF Statistics, In: D' AGOSTINO, R. B.; STEPHENS, M. A. (Eds.) Goodness-of-Fit Techniques, Marcel Dekker, New York, pp. 97-193.

YILMAZ, V.; ÇELIK, H. E. 2008. A statistical approach to estimate the wind speed distribution: the case of Gelibolu region.

ZAHARIM, A.; RAZALI, A. M.; ABIDIN, R. Z.; SOPIAN, K. 2009. Fitting of statistical distributions to wind speed data in Malaysia. European Journal of Scientific Research, v. 26, n. 1, p. 6-12. 